Paradoxes and counterexamples in teaching and learning of probability at university

Sergiy Klymchuk and Farida Kachapova

School of Computing and Mathematical Sciences, Auckland University of Technology, Auckland, New Zealand

Corresponding author:

Dr Sergiy Klymchuk

Associate Professor

School of Computing and Mathematical Sciences

Faculty of Design and Creative Technologies

Auckland University of Technology

Private Bag 92006

Auckland 1142

New Zealand

Tel: +64-9-921 9999 ext.8431

Fax: +64-9-921 9944

E-mail: sergiy.klymchuk@aut.ac.nz 


\section{Paradoxes and counterexamples in teaching and learning of probability at university}

The paper is devoted to practical aspects of teaching and learning of probability at university. It presents the difficulties and attitudes of first-year university science and engineering students towards using paradoxes and counterexamples as a pedagogical strategy in teaching and learning of probability. It also presents a student's point of view on the effectiveness of this pedagogical strategy.

Keywords: paradoxes; counterexamples; probability; teaching; learning

\section{Introduction}

Several studies investigated the usage of paradoxes on students' motivation of learning probability and statistical concepts [1-6]. Lesser [6] made a connection between so called counterintuitive results in probability and statistics and statistical literacy. He gave an example that 'Simpson's Paradox is listed as essential for citizenship by the National Council on Education and the Disciplines [7]' [6]. Several books are written on counterexamples in probability and statistics [8-10]. A number of studies about the effect of available counterexamples on conditional inference making were reviewed in [11]. In this paper, we present a student's point of view on the effectiveness of the usage of paradoxes and counterexamples as a pedagogical strategy in the teaching and learning of probability in a first-year university course.

\section{The study}

\subsection{Participants}

Two groups of students were exposed to the practice of using paradoxes and counterexamples as a pedagogical strategy in teaching and learning of probability. The first group comprised 11 students from [name deleted to maintain the integrity of the review process] studying a first-year course 'Probability Theory and Applications'. The students from the first group were majoring in science and applied mathematics. The second group comprised 30 students from [name deleted to maintain the integrity of the review process] studying probability 
theory as part of their first-year mathematics course. The students from the second group were majoring in engineering. At the end of the semester the students were given a questionnaire to investigate their attitudes towards the usage of paradoxes and counterexamples in teaching and learning of probability. All 11 students from the first group and 23 students out of 30 from the second group answered the questionnaire. The students in the German university received the questionnaire in German. The students' responses in both groups were very similar, so we combined them. The response rate therefore was 34/41, which is $83 \%$. The participation in the study was voluntary, so we had a self-selected sample.

\subsection{Using paradoxes and counterexamples in teaching}

In our study we did not use 'pathological' cases. All paradoxes and incorrect statements for creating counterexamples that were given to the students were within their knowledge and often related to their common misconceptions. Paradoxes and incorrect statements were given to the students for a group discussion after solving a number of routine exercises on a topic. The intention was to enhance the students' understanding of probability concepts, increase their motivation to study the subject and engage their emotions as both paradoxes and counterexamples were non-routine exercises with counterintuitive and surprising answers. Paradoxes were not included in the assessment. Exercises on creating counterexamples to incorrect statements were included in home assignments that were part of the assessment.

Below are some of the paradoxes that were discussed with the students with the lecturers' comments and hints that helped the students to resolve them.

Galton's paradox. [12, p. 14]. 
You flip three fair coins. At least two results are alike (the same). There is 50-50 chance that the third one is a head or a tail. Therefore the probability that all three results are alike equals 0.5. Do you agree?

Most students agreed with the suggested wrong answer. It was hard for them to discover a flaw in this argument because they did not analyse the problem mathematically. The lecturer reminded them that in the current lesson they studied probability spaces and suggested constructing one for this experiment. The students had already learned several examples with coin tosses, so they successfully wrote down the sample space for the experiment:

$$
\Omega=\{H H H, \text { HHT, HTH, HTT, THH, THT, TTH, TTT }\} \text {, }
$$

where $\mathrm{H}$ stands for head and T for tail. With the lecturer's help, the students concluded that all eight outcomes are equiprobable because the coins are fair (symmetrical). They presented the event $\mathrm{A}=$ "all three results are the same" in terms of the outcomes: $\mathrm{A}=\{\mathrm{HHH}, \mathrm{TTT}\}$.

Since A contains two outcomes, its probability was calculated as $2 \times \frac{1}{8}=\frac{1}{4}$.

Although the students had enough knowledge to solve the problem correctly, at the beginning they did not apply their knowledge and just accepted the flawed solution.

Simpson's paradox. [12, p. 19].

A doctor has performed clinical trials to determine the relative efficacies of two drugs, with the results presented in Table 1 below:

(Table 1. Results of drug treatment.)

\begin{tabular}{|l|c|c|c|c|}
\hline \multirow{2}{*}{} & \multicolumn{2}{|c|}{ Women } & \multicolumn{2}{c|}{ Men } \\
\cline { 2 - 5 } & Drug 1 & Drug 2 & Drug 1 & Drug 2 \\
\hline Success & 200 & 10 & 19 & 1000 \\
\hline Failure & 1800 & 190 & 1 & 1000 \\
\hline Total & 2000 & 200 & 20 & 2000 \\
\hline
\end{tabular}

a) Calculate the overall success rate of each drug. 
b) Calculate the success rate of each drug for men.

c) Calculate the success rate of each drug for women.

d) Which drug is better?

The students had no difficulties in answering questions a) - c).

a) The overall success rate equals $\frac{200+19}{2000+20} \approx 0.108$ for Drug 1 and $\frac{10+1000}{200+2000} \approx 0.459$ for Drug 2

b) The success rate for men equals $\frac{19}{20}=0.95$ for Drug 1 and $\frac{1000}{2000}=0.5$ for Drug 2 .

c) The success rate for women equals $\frac{200}{2000}=0.1$ for Drug 1 and $\frac{10}{200}=0.05$ for

\section{Drug 2.}

They had a group discussion, which showed that they were puzzled by the results of their calculations: Drug 1 has better results when men and women are considered separately but Drug 2 has a better result when the two groups are combined. The students' intuition said that the same drug should work better in all cases. Some of the students said: "perhaps my calculations were wrong”. They could not reach a common conclusion for question d). The lecturer commented on the importance of causal relationships, which helped the students to realise that Drug 1 was better (since it had higher success rates for men and women separately). Then the students discussed other cases when the Simpson's paradox might arise. We observed an increased interest and motivation to the subject in both New Zealand and German groups of students when we mentioned the fact that Simpson's paradox is considered so important for statistical literacy in the USA that it is listed as essential for citizenship by the National Council on Education and the Disciplines [7].

Monty Hall paradox. [12, p. 12]. 
Suppose you are in a game show, and you are given the choice of three doors of which one contains a prize. The other two contain gag gifts like a goat or a donkey. You pick a door, say, No. 1. The host (who knows what behind the doors) opens door 3, which has a donkey. He then says to you, “Do you want to pick door 2?”. Is it to your advantage to switch your choice? That is, will your probability of winning increase if you switch to door 2 ?

Most students answered that switching to door 2 would not increase the probability of winning (as their intuition said). The lecturer recommended using a probability tree and calculating conditional probabilities. By the way, we observed that probability trees were the students' preferred tool for solving problems about conditional probabilities; they mastered this technique faster and used it more successfully than two-way tables or the partition theorem. For the probability tree in the Monty Hall paradox some students needed help with identifying the two main hypotheses: "the prize is behind door 1" and "the prize is behind door 2”. After that they were able to construct the probability tree shown in Figure 1 below:

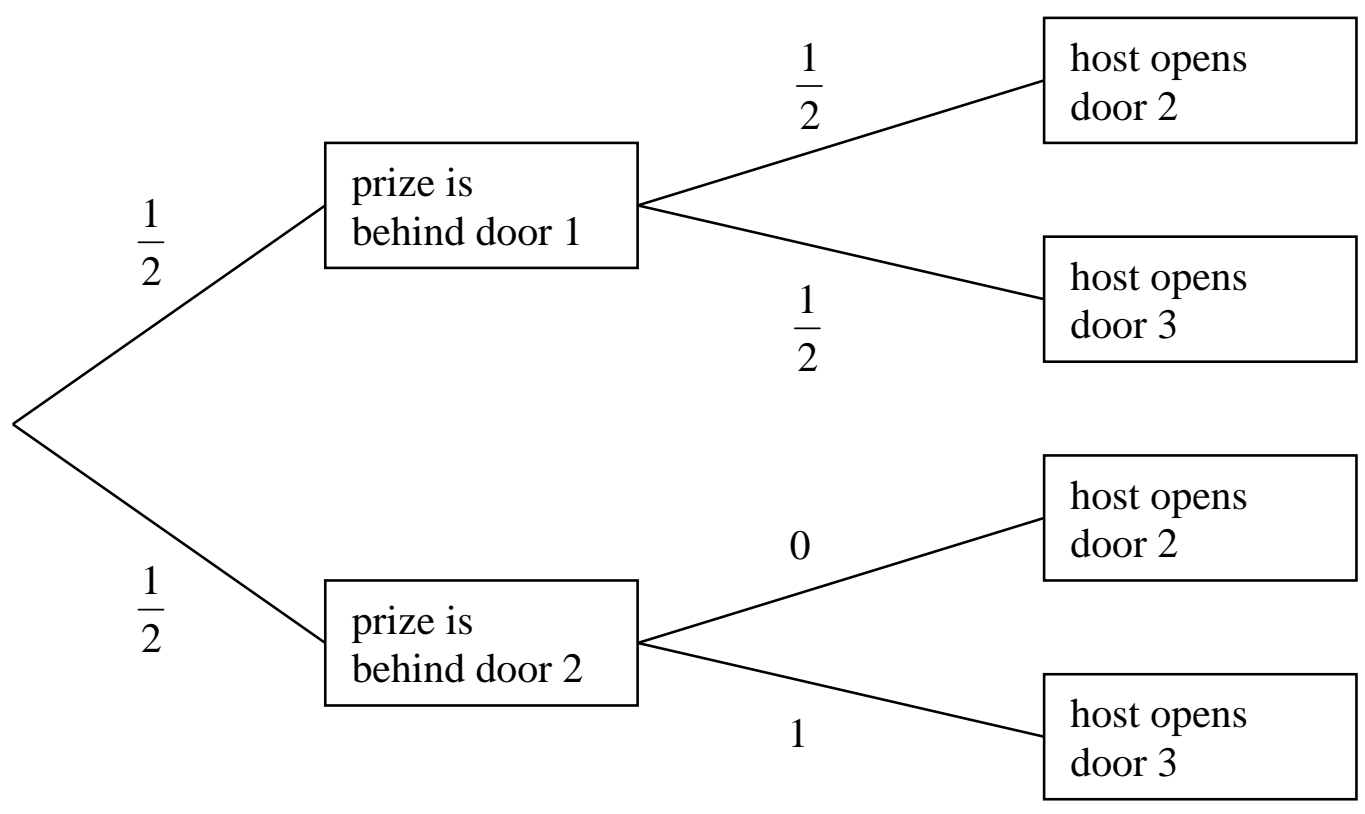

(Figure 1. Probability tree for the Monty Hall paradox.) 
Since the students had already mastered the technique of probability trees, they easily evaluated the probability of the host opening door 3 as $\frac{1}{2} \times \frac{1}{2}+\frac{1}{2} \times 1=\frac{3}{4}$ and obtained these conditional probabilities:

$$
\begin{aligned}
& \mathrm{P} \text { (prize is behind door } 1 \mid \text { host opens door } 3)=\frac{\frac{1}{2} \times \frac{1}{2}}{\frac{3}{4}}=\frac{1}{3} \\
& \text { and P (prize is behind door } 2 \mid \text { host opens door } 3 \text { ) }=\frac{\frac{1}{2} \times 1}{\frac{3}{4}}=\frac{2}{3} \text {. }
\end{aligned}
$$

This led them to a surprising (but correct) answer: "the probability of winning is twice higher if you switch to door 2”.

There was a group discussion of the result, and in particular of the importance of stating the problem in exact terms (here - in terms of events) and using the mathematical concept of conditional probability. After the lecturer's suggestion, the students also discussed how the answer depends on whether the host does or does not know what is behind the doors.

Prisoners’ paradox. [12, p. 11].

There are three prisoners, $A, B$, and $C$. The warden tells them that two of them will be released and one will be executed. But he is not permitted to reveal to any prisoner the fate of that prisoner.

A asks the warden to tell him the name of one of his cohort who will be released. The warden obliges and says, " $B$ will be released." Assume that the warden tells the truth.

a) What are A's and C's respective probabilities of dying now?

b) If A could switch fates with $C$ now, should he?

As in the previous cases, most students used their intuition and gave wrong answers. It is understandable, since paradoxes are counterintuitive. The lecturer reminded the students that they needed to apply mathematical techniques and pointed out the similarity of this paradox with the Monty Hall paradox. Then many students tried to imitate the probability 
tree from the previous paradox and subsequently obtained wrong answers. Only two students successfully constructed the appropriate probability tree shown in Figure 2 below:

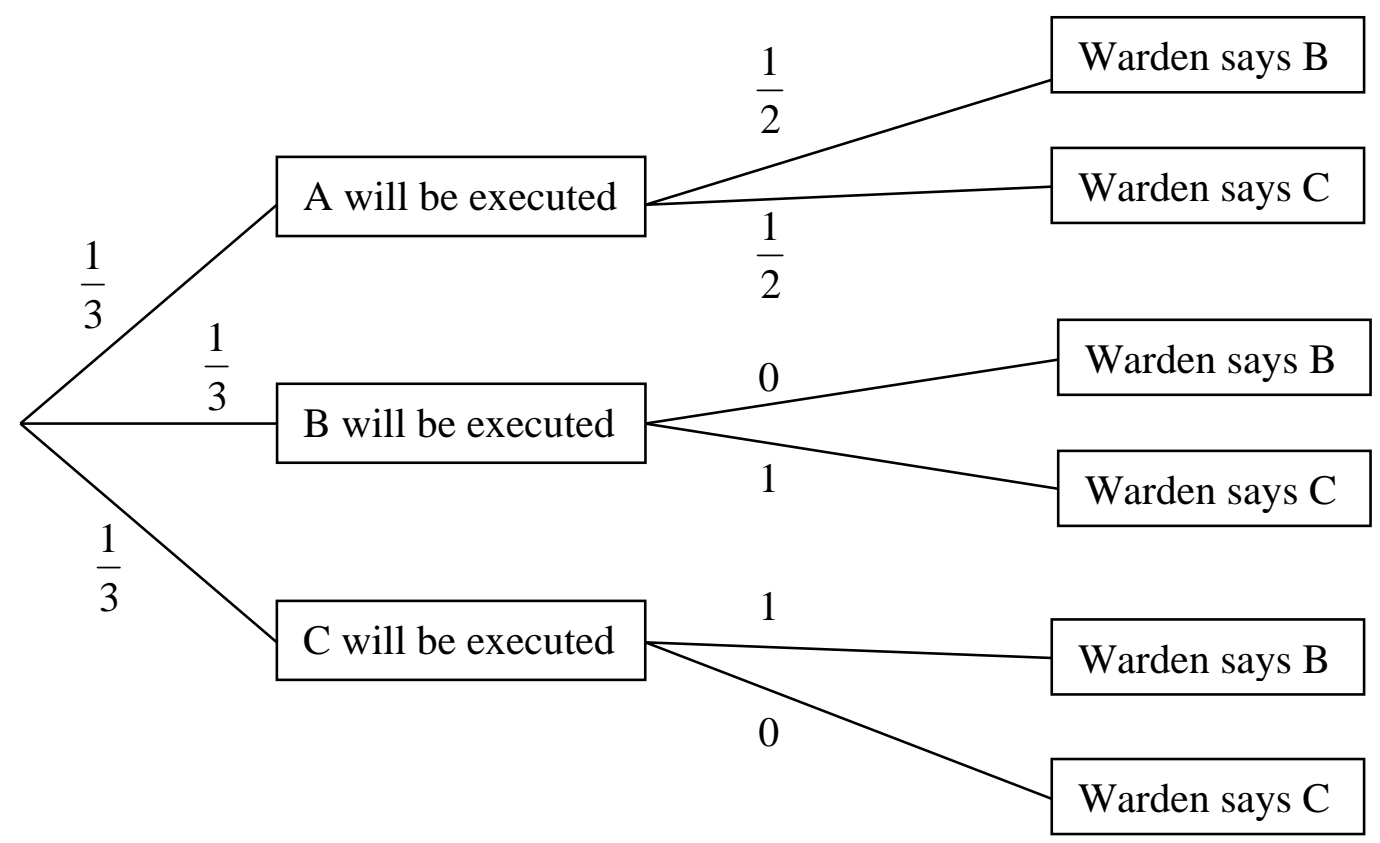

(Figure 2. Probability tree for the Prisoners’ paradox.)

This tree was presented to all the students for a group discussion, and they used it to correctly calculate the A's and C' respective probabilities of dying as $\frac{1}{3}$ and $\frac{2}{3}$.

St Petersburg's paradox. [12, p. 55].

In a game of chance, a player pays a fixed fee to enter, and then a fair coin is tossed repeatedly until a head appears ending the game. If the first head appears after $n$ tosses, then the player gets $\$ 2^{n}$.

a) What is the expected win of a player?

b) What is the "fair" entrance fee?

Though all the students had done a calculus course before, half of them had difficulties producing and analysing the infinite series in question a). The other half gave the correct answer "infinity" but could not explain this result or answer question b). The lecturer 
explained that the paradox can be resolved by assigning more weight to loss of money than its gain, and the students' intuition seemed to agree with this practical approach.

Apart from paradoxes we also used counterexamples in teaching the courses. Below are examples of incorrect statements that the students had to disprove by creating counterexamples.

Statement 1. Pairwise independence of events implies their mutual independence.

Statement 2. If events $A$ and $B$ are independent, then they are conditionally independent.

Statement 3. If events $A$ and $B$ are conditionally independent, then they are independent.

Statement 4. Uncorrelated random variables are independent.

Statement 5. Pairwise independence of random variables implies their mutual independence.

The notion of a counterexample was discussed with the students as many of them had never heard about it before. In the beginning the students had difficulties in creating counterexamples, so some guidance was needed from the lecturer. For example, for Statement 4 the lecturer introduced the joint distribution of random variables $X$ and $Y$ presented in Table 2 below:

(Table 2. Lecturer's counterexample to Statement 4.)

\begin{tabular}{|c|c|c|c|}
\hline $\boldsymbol{Y}$ & 0 & 1 & 2 \\
\hline 0 & $\frac{1}{4}$ & 0 & $\frac{1}{4}$ \\
\hline 1 & 0 & $\frac{1}{2}$ & 0 \\
\hline
\end{tabular}

Then the students verified that $X$ and $Y$ make a counterexample to Statement 4 . They had a group discussion of the result and were asked to make their own counterexamples. A successful student's attempt is presented in Table 3 below: 
(Table 3. Student's counterexample to Statement 4.)

\begin{tabular}{|c|c|c|c|}
\hline $\boldsymbol{Y}$ & -1 & 0 & 1 \\
\hline-1 & $\frac{1}{3}$ & 0 & $\frac{1}{3}$ \\
\hline 1 & 0 & $\frac{1}{3}$ & 0 \\
\hline
\end{tabular}

\subsection{The questionnaire}

The students' attitudes towards the usage of paradoxes and counterexamples in teaching and learning of probability were investigated using the following questionnaire.

Question 1.

Do you find paradoxes and counterexamples useful for understanding this course?
a) Yes
Please give the reasons:
b) No
Please give the reasons:

Question 2.

Do you feel confident using paradoxes and counterexamples?
a) Yes
Please give the reasons:
b) No
Please give the reasons:

Question 3.

Do you find this strategy effective?
a) Yes
Please give the reasons:
b) No
Please give the reasons:

Question 4.

Would you like this kind of activity to be a part of assessment?
a) Yes
Please give the reasons:
b) No
Please give the reasons: 


\subsection{Findings from the questionnaire}

The statistics from the questionnaire are presented in Table 4 below:

(Table 4. Summary of findings from the questionnaire.)

\begin{tabular}{|c|c|c|c|c|c|c|c|c|}
\hline \multirow{2}{*}{ Number of Students } & \multicolumn{2}{|c|}{$\begin{array}{c}\text { Question 1 } \\
\text { Useful? }\end{array}$} & \multicolumn{2}{c|}{$\begin{array}{c}\text { Question 2 } \\
\text { Confident? }\end{array}$} & \multicolumn{2}{c|}{$\begin{array}{c}\text { Question 3 } \\
\text { Effective? }\end{array}$} & \multicolumn{2}{c|}{$\begin{array}{c}\text { Question 4 } \\
\text { Assessment? }\end{array}$} \\
\cline { 2 - 10 } & Yes & No & Yes & No & Yes & No & Yes & No \\
\hline 34 & 31 & 3 & 16 & 18 & 30 & 4 & 11 & 23 \\
\hline Percentage & $91 \%$ & $9 \%$ & $47 \%$ & $53 \%$ & $88 \%$ & $12 \%$ & $32 \%$ & $68 \%$ \\
\hline
\end{tabular}

The vast majority of the students reported that they found the method of using

paradoxes and counterexamples to be useful (91\%) and effective (88\%). The common

comments from the students who answered 'Yes' to question 1 on the usefulness and

question 3 on the effectiveness were as follows:

'They are both entertaining and informative; they are helpful because we can look back at them when we do assignments; we go through reasoning of counterexamples and paradoxes that helps understanding the course; helps to overcome superficial knowledge; good illustration of theory; it makes the use of probabilities more clear; examples explain complex connections; it builds my logical skills; motivates to be critical and to overcome stupid solution schemes; trains to reconsider - paradoxes are more difficult than counterexamples; it supports deeper thinking and understanding; examples are illustrative and interesting and good for logical thinking; they support the understanding of problems; it strengthens my thinking ability; it is interesting to consider different solutions; better understanding; it improves my understanding of probability and random variables; helps understanding and logical thinking; better feeling (sense) for the topic; paradoxes at first look confusing, but then revealing; different aspects are shown and broaden knowledge; promotes the understanding which is useful for the future; this teaching principle was very helpful for me; the illustration helps in understanding the whole; supports interest and deep thinking; encourage to think'.

About half (47\%) of the students eventually felt confident using paradoxes and counterexamples and the others (53\%) did not. More than 2/3 of the students (68\%) did not like the idea of using paradoxes and counterexamples in assessment. The main reasons indicated by the students were the time factor ('counterexamples and paradoxes are difficult to solve under time pressure', 'too complex and time consuming for examinations') and the 
stress in the exam situation ('for most students normal maths is stressful enough, weak students would be desperate’, 'under examination stress we behave rationally, and paradoxes are against rationality'). To some extent this contradicts the responses to questions 1 and 3, where the vast majority of the students indicated that they found paradoxes and counterexamples useful and effective. Clearly the students were more concerned about their exam performance rather than acquiring useful skills.

\section{Conclusions and Recommendations}

The statistics results and numerous students’ comments from the study showed that the students were very positive about the usage of paradoxes and counterexamples in teaching and learning of probability. The vast majority of them reported that they found this pedagogical strategy to be useful and effective. Thirty six percent of the students made the comment that this pedagogical strategy helped them to improve their logical thinking. As the first step in this teaching approach we would recommend that a lecturer provides a paradox or a counterexample and asks students to explain or justify it. Next the students could be asked to create their own counterexamples to a given incorrect statement. And finally, the lecturer can ask the students to decide whether a given mathematical statement is correct, so the students have to come up with a proof that the statement is true, or with a counterexample to show that the statement is false. There are many ways of using counterexamples as a pedagogical strategy:

- giving students a mixture of correct and incorrect statements;

- asking students to create their own wrong statements and counterexamples to them;

- making a deliberate mistake in a lecture;

- $\quad$ asking students to spot an error on a certain page of their textbook; 
- giving students bonus marks towards their final grade for creating excellent counterexamples;

- including into assessment the questions that require construction of counterexamples.

In a one-semester course we tried to lead the students through some of these activities with a certain amount of success. From the students' very positive feedback on the usefulness (91\%) and effectiveness (88\%) of the usage of paradoxes and counterexamples in teaching and learning of probability it is clear that not only top students but the majority of average and weak students were enthusiastic about studying paradoxes and counterexamples in the probability course. It gives us confidence to recommend this pedagogical strategy to our colleagues who teach probability in a first-year university course.

\section{Acknowledgements}

We would like to express our sincere gratitude to our colleagues [names deleted to maintain the integrity of the review process], for translating their students' responses into English.

\section{References}

[1] J.M. Shaughnessy, Misconceptions of Probability: An Experiment with a Small Group, Activity-Based, Model Building Approach to Introductory Probability at the College Level, Educ. Stud. Math. 8 (1977), pp. 285-316.

[2] N. Movshovitz-Hadar and R. Hadass, Preservice Education of Math Teachers Using Paradoxes, Educ. Stud. Math. 21 (1990), pp. 265-287.

[3] U. Wilensky, Paradox, Programming and Learning Probability: A Case Study in a Connected Mathematics Framework. J. of Math. Behavior 14 (1995), pp. 253-280.

[4] E.R. Sowey, Striking demonstrations in teaching statistics, J. of Stat. Ed. 9 (1) (2001). Available at http://www.amstat.org/publications/jse/v9n1/sowey.html.

[5] T. Leviatan, On the use of paradoxes in the teaching of probability, in Proceedings of the Sixth International Conference on Teaching Statistics (ICOTS 6), South Africa, 2002.

[6] L.M. Lesser, Engaging the intuition in statistics to motivate, AP Statistics Course, Home Page at College Board, AP Central, (2006). Available at http://apcentral.collegeboard.com/apc/members/courses/teachers_corner/50025.html

[7] National Council on Education and the Disciplines, Mathematics and Democracy: The Case for Quantitative Literacy. Woodrow Wilson National Fellowship Foundation, Princeton, New Jersey, 2001.

[8] J.P. Romano and A.F. Siegel, Counterexamples in Probability and Statistics. Chapman and Hall/CRC, Boca Raton, 1986. 
[9] J.L. Wise and E.B. Hall, Counterexamples in Probability and Real Analysis, Oxford University Press, New York, 1993.

[10] J.M. Stoyanov, Counterexamples in Probability, $2^{\text {nd }}$ ed., Wiley, England, 1997.

[11] G. Politzer and G. Bourmaud, Deductive reasoning from uncertain conditionals. British J. of Psychology, 93 (2002), pp. 345 - 981.

[12] G. Grimmett and D. Stirzaker, Probability and Random Processes, $3^{\text {rd }}$ ed., Oxford University Press, New York, 2004. 